\title{
Polarized micro-Raman studies of femtosecond laser written stress-induced optical waveguides in diamond
}

\author{
B. Sotillo, ${ }^{1, a)}$ A. Chiappini, ${ }^{2}$ V. Bharadwaj, ${ }^{1}$ J. P. Hadden, ${ }^{3}$ F. Bosia,${ }^{4}$ P. Olivero, ${ }^{4}$ M. Ferrari, ${ }^{2}$ \\ R. Ramponi, ${ }^{1}$ P. E. Barclay, ${ }^{3}$ and S. M. Eaton ${ }^{1}$ \\ ${ }^{1}$ Istituto di Fotonica e Nanotecnologie-Consiglio Nazionale delle Ricerche (IFN-CNR) and Department \\ of Physics, Politecnico di Milano, Piazza Leonardo da Vinci 32, 20133 Milano, Italy \\ ${ }^{2}$ Istituto di Fotonica e Nanotecnologie-Consiglio Nazionale delle Ricerche (IFN-CNR) Characterization and \\ Development of Materials for Photonics and Optoelectronics (CSMFO) and The Centre for Materials and \\ Microsystems (FBK-CMM), 38123 Trento, Italy \\ ${ }^{3}$ Institute for Quantum Science and Technology, University of Calgary, AB T2N 1N4 Calgary, Canada \\ ${ }^{4}$ Department of Physics and "Nanostructured Interfaces and Surfaces" Inter-Departmental Centre, \\ University of Torino, I-10125 Torino, Italy
}

(Received 24 November 2017; accepted 30 December 2017; published online 17 January 2018)

\begin{abstract}
Understanding the physical mechanisms of the refractive index modulation induced by femtosecond laser writing is crucial for tailoring the properties of the resulting optical waveguides. In this work, we apply polarized Raman spectroscopy to study the origin of stress-induced waveguides in diamond, produced by femtosecond laser writing. The change in the refractive index induced by the femtosecond laser in the crystal is derived from the measured stress in the waveguides. The results help to explain the waveguide polarization sensitive guiding mechanism, as well as provide a technique for their optimization. Published by AIP Publishing. https://doi.org/10.1063/1.5017108
\end{abstract}

Color centers in diamond, such as nitrogen-vacancy (NV) or silicon-vacancy (SiV) centers, show great potential for quantum systems, ${ }^{1}$ temperature sensing, ${ }^{2,3}$ or magnetic field sensing in the case of the NV center. ${ }^{4}$ These defects can be initialized, manipulated, and read out using photons. Optical waveguides in bulk diamond could be used to optically link and address these color centers. ${ }^{5}$ Several methods have been used for fabricating waveguides in diamond, such as ion beam assisted lift-off, ${ }^{6}$ plasma etching, ${ }^{7,8}$ or ion implantation. ${ }^{9}$ Recently, optical waveguides ${ }^{10,11}$ and Bragg gratings ${ }^{12}$ in bulk diamond have been formed using the femtosecond laser technique, opening the possibility of creating $3 \mathrm{D}$ photonic circuits in this material. A deeper understanding of the physics underlying the writing of optical waveguides in diamond will help in the development of advanced devices integrating photonics circuits and color centers. ${ }^{13}$

Femtosecond laser writing relies on the nonlinear absorption of focused ultrashort pulses, which leads to a localized modification in the bulk of transparent materials. ${ }^{14-16}$ In crystals such as diamond, laser interaction typically produces a decrease in the refractive index due to the damage of the lattice, and so, the strategy for fabricating the waveguides is to write two lines separated by several microns and confine the optical mode between these barriers. ${ }^{10,17}$ In such waveguides, the increase in the refractive index is associated with the stress induced between the two lines due to the volume variation of the material inside the laser-written modifications. ${ }^{18}$

Micro-Raman spectroscopy has been previously applied to study the waveguides formed in different crystals. ${ }^{19-21}$ However, to date, no works have managed to describe the relationship between the polarized Raman signal, the stress induced in the waveguides, and its relation with the change in the refractive index. Refracted near field profilometry is

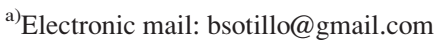

the only non-destructive measurement technique for direct quantitative characterization of the cross-sectional refractive index profile of bulk waveguides. ${ }^{22,23}$ However, this technique requires index matching oil (maximum refractive index 1.8), incompatible with the refractive index of diamond (2.4). Although the quantitative phase microscopy method would allow the measurement of the refractive index change in waveguides within high refractive index materials, ${ }^{24}$ its complexity and destructive nature make it undesirable for the characterization of diamond. On the other hand, confocal polarized $\mu$ Raman spectroscopy is a non-destructive technique with a micrometer spatial resolution and is sensitive to the local stresses present in the material and thus will greatly benefit the understanding of the stress-induced waveguides in diamond, as well as in other crystalline materials.

The diamond samples used in this work were synthetic CVD grown single crystal diamond, type IIa, with a dimension of $5 \times 5 \times 0.5 \mathrm{~mm}^{3}$. They were purchased from $\mathrm{MB}$ optics, with all the facets polished and with an orientation of $4 \mathrm{pt}$ ( $\{100\}$-planes) for the top and bottom larger surfaces and $2 \mathrm{pt}$ ( $\{110\}$-planes) for the side surfaces. If we define the crystal axis system as $\mathrm{X}=[100], \mathrm{Y}=[010]$ and $\mathrm{Z}=[001]$ [Fig. 1(a)], the coordinate system for the sample will be defined as $\mathrm{X}^{\prime}=[110], \mathrm{Y}^{\prime}=[\overline{1} 10]$, and $\mathrm{Z}^{\prime}=[001]$, as shown in Fig. 1(b), with $X^{\prime} Y^{\prime} Z^{\prime}$ being obtained by a $45^{\circ}$ rotation of the $X Y Z$ coordinates around the $\mathrm{Z}$ axis.

The femtosecond laser used for writing optical waveguides in diamond was a regeneratively amplified $\mathrm{Yb}: \mathrm{KGW}$ system (Pharos, Light Conversion) with a 230-fs pulse duration and a 515-nm wavelength, focused with a 1.25-NA oil immersion lens (RMS100X-O 100× Olympus Plan Achromat Oil Immersion Objective). The repetition rate of the laser was $500 \mathrm{kHz}$, and the pulse energy was $100 \mathrm{~nJ}$. Computercontrolled, 3-axis motion stages (ABL-1000, Aerotech) were used to translate the sample relative to the laser with a scan 


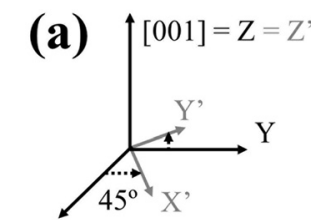

$[100]=X$

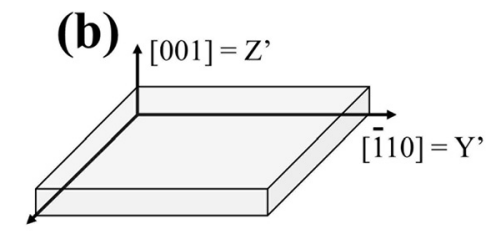

$[110]=X^{\prime}$
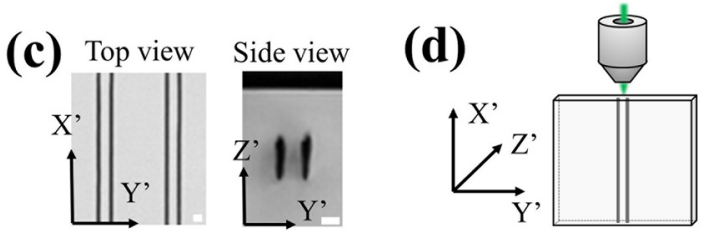

FIG. 1. Orientation of the diamond samples examined. (a) Crystal axis system; (b) sample axis system. (c) Optical micrographs of the diamond waveguide (scale bar of $10 \mu \mathrm{m}$ ). (d) Sample orientation in the Raman setup.

speed of $0.5 \mathrm{~mm} / \mathrm{s}$. In the $\mathrm{X}^{\prime} \mathrm{Y}^{\prime} \mathrm{Z}^{\prime}$ coordinate system shown in Fig. 1(b), the laser is incident along the $[00 \overline{1}]$ direction, the modification lines are formed along the [110] direction, and the linear polarization of the laser is along the $[\overline{1} 10]$ direction. Overhead and transversal microscopy images (obtained with a Nikon Eclipse ME600 microscope) of the laser-written lines are shown in Fig. 1(c), indicating also the $X^{\prime} Y^{\prime} Z^{\prime}$ coordinate system. To measure the near-field waveguide optical mode profile, light was coupled to the waveguides using a single-mode fiber and the output was imaged with a $60 \times$ aspheric lens (5721-H-B, Newport) on a beam profiler (SP620U, Spiricon). A mode field diameter of $10 \mu \mathrm{m}$ was measured for a wavelength of $635 \mathrm{~nm}$ for lines separated by $13 \mu \mathrm{m}$. Further details of the waveguide characterization can be found in Ref. 10 .

Finally, $\mu$ Raman spectra were recorded using a Labram Aramis Jobin Yvon Horiba microRaman system with a DPSS laser source of $532 \mathrm{~nm}$ and equipped with a confocal microscope and an air-cooled CCD. The pinhole was $300 \mu \mathrm{m}$. A $100 \times$ objective $(0.8 \mathrm{NA})$ was used to focus the laser on the (110) plane of the sample as well as to collect the Raman signal (backscattering configuration), as shown in Fig. 1(d). This experimental configuration allows us to detect the Raman modes needed to perform this study. The spatial resolution is below $1 \mu \mathrm{m}$.

A cubic diamond lattice (space group $O_{h}$ ) has three optical phonons with $F_{2 g}$ symmetry at the center of the Brillouin zone. These phonons, in the absence of strain in the lattice, have the same frequency $\left(\omega_{0}=1333 \mathrm{~cm}^{-1}\right)$. The Raman intensity collected is given by the following expression, which depends on the Raman tensors $\left(R_{j}\right)$ and the polarization vectors of the incident $\left(e_{i}\right)$ and scattered $\left(e_{s}\right)$ light:

$$
I \propto \sum\left|e_{i} \cdot R_{j} \cdot e_{s}\right|^{2}
$$

The Raman tensors at the center of the Brillouin zone in the $\mathrm{XYZ}$ crystal system are

$$
\begin{aligned}
& R_{1}=\left(\begin{array}{lll}
0 & 0 & 0 \\
0 & 0 & d \\
0 & d & 0
\end{array}\right) ; \quad R_{2}=\left(\begin{array}{lll}
0 & 0 & d \\
0 & 0 & 0 \\
d & 0 & 0
\end{array}\right) ; \\
& R_{3}=\left(\begin{array}{lll}
0 & d & 0 \\
d & 0 & 0 \\
0 & 0 & 0
\end{array}\right),
\end{aligned}
$$

where $d$ accounts for the variation of the polarizability with the displacement of the atoms in the lattice. ${ }^{25}$ For the coordinate system of our diamond sample $X^{\prime} Y^{\prime} Z^{\prime}$, these tensors will be transformed as (rotation of $45^{\circ}$ around the $Z$ axis)

$$
\begin{aligned}
& R_{1}^{\prime}=d\left(\begin{array}{ccc}
0 & 0 & -1 / \sqrt{2} \\
0 & 0 & 1 / \sqrt{2} \\
-1 / \sqrt{2} & 1 / \sqrt{2} & 0
\end{array}\right) ; \\
& R_{2}^{\prime}=d\left(\begin{array}{ccc}
0 & 0 & 1 / \sqrt{2} \\
0 & 0 & 1 / \sqrt{2} \\
1 / \sqrt{2} & 1 / \sqrt{2} & 0
\end{array}\right) ; \quad R_{3}^{\prime}=d\left(\begin{array}{ccc}
-1 & 0 & 0 \\
0 & 1 & 0 \\
0 & 0 & 0
\end{array}\right) .
\end{aligned}
$$

It is well known that when stress is applied to the diamond crystal, the cubic symmetry is removed and the degeneracy of the three optical phonons is lifted. ${ }^{26-31}$ In order to obtain the new frequencies of the phonons at the zone-center related to the applied stress, the following secular equation which is derived from lattice dynamical equations ${ }^{26}$ has to be solved:

$$
\left|\begin{array}{ccc}
p \epsilon_{1}+q\left(\epsilon_{2}+\epsilon_{3}\right)-\lambda & r \epsilon_{6} & r \epsilon_{5} \\
r \epsilon_{6} & p \epsilon_{2}+q\left(\epsilon_{1}+\epsilon_{3}\right)-\lambda & r \epsilon_{4} \\
r \epsilon_{5} & 2 r \epsilon_{4} & p \epsilon_{3}+q\left(\epsilon_{1}+\epsilon_{2}\right)-\lambda
\end{array}\right|=0
$$

where $p, q$, and $r$ are the deformation potential constants for diamond $^{27,31}\left(p=-2.810 \omega_{0}^{2} ; q=-1.77 \omega_{0}^{2} ; r=-1.990 \omega_{0}^{2}\right)$ and the eigenvalues $\lambda_{i}$ are related to the former $\left(\omega_{0}\right)$ and new frequencies of the three modes $\left(\omega_{i}\right):{ }^{29} \lambda_{i}=\omega_{i}^{2}-\omega_{0}^{2} ; \Delta \omega_{i}$ $=\omega_{i}-\omega_{0} \approx \frac{\lambda_{i}}{2 \omega_{0}}$. $\epsilon$ represents the components of the strain matrix, which are related to the stress $\sigma$ by Hooke's law: $\epsilon_{i}=S_{i j} \sigma_{j}\left(\mathrm{i}, \mathrm{j}=1, \ldots, 6\right.$, with $S_{i j}$ being the compliance constants, following the matrix notation ${ }^{32}$ ).
For a cubic crystal, due to symmetry considerations, only three components are non-zero, having the following values for diamond: ${ }^{31} S_{11}=0.952 \times 10^{-3} \mathrm{GPa} ; S_{12}=-0.099$ $\times 10^{-3} \mathrm{GPa} ; S_{44}=1.737 \times 10^{-3} \mathrm{GPa}$. As noted above, in the selected scenario of our waveguide, we are going to study the Raman signal in the backscattering configuration from the (110) surface, considering that the stress produced by the two laser-written tracks has biaxial character in the (110) 
plane. The stress matrix referred to the $X^{\prime} Y^{\prime} Z^{\prime}$ system is then defined as

$$
\sigma^{\prime}=\left(\begin{array}{ccc}
0 & 0 & 0 \\
0 & \tau_{1} & 0 \\
0 & 0 & \tau_{2}
\end{array}\right),
$$

where $\tau_{1}$ is the stress parallel to the [110] direction and $\tau_{2}$ is the stress parallel to the [001] direction. This matrix can be rotated into the $\mathrm{XYZ}$ crystal coordinates by applying a rotation of $-45^{\circ}$ around the $\mathrm{Z}^{\prime}=\mathrm{Z}$ axis

$$
\sigma=\left(\begin{array}{ccc}
\tau_{1} / 2 & \tau_{1} / 2 & 0 \\
\tau_{1} / 2 & \tau_{1} / 2 & 0 \\
0 & 0 & \tau_{2}
\end{array}\right) .
$$

Using this matrix, the strain components are calculated using Hooke's law: $\epsilon_{1}=\epsilon_{2}=s_{12} \tau_{2}+\frac{\tau_{1}}{2}\left(s_{11}+s_{12}\right) ; \epsilon_{3}=s_{11} \tau_{2}$ $+s_{12} \tau_{1} ; \epsilon_{6}=\frac{s_{4} \tau_{1}}{2}$. By substituting in secular Eq. (2), the eigenvalues are obtained

$$
\begin{aligned}
\Delta \omega_{1} & =-\left(0.82 \tau_{2}+0.085 \tau_{1}\right), \\
\Delta \omega_{2} & =-\left(0.82 \tau_{2}+2.28 \tau_{1}\right), \\
\Delta \omega_{3} & =-\left(1.55 \tau_{2}+0.82 \tau_{1}\right) .
\end{aligned}
$$

In the case of $\tau_{1}=\tau_{2}$, the values reported by Von Kaenel et $a l .{ }^{29}$ are reproduced. These results indicate that the applied stress lifted completely the triple degeneracy. Note that the sign convention for stress is that tensile stresses are positive while compressive are negative. From Eq. (1) and considering the conservation of the wavevector, we can derive the components that will be visible for backscattering from a (110) surface, which are shown in Table I, in agreement with other studies ${ }^{29,33}$ (note that Porto's notation is used ${ }^{34}$ ).

From previous studies, ${ }^{28}$ the out-of-plane component will show the most different value of shift. So, the values obtained in Eq. (5) will be related to this component, whereas Eqs. (6) and (7) are the components that will be measured in our experiments $(/ /[001]$ and $/ /[\overline{1} 10]$ components).

In Fig. 2, the maps for the intensity and the shift of the diamond peak (from the fitting to Lorentzian curves) are shown. The Raman peak shows an intensity decrease in the laser-written tracks associated with disorder and change in the $\mathrm{sp}^{2}$-like phase induced by the laser. ${ }^{10}$ The results obtained within the modified laser regions are not physically meaningful in this study as the fit of the diamond peak is more complex due to the presence of disorder and non-diamond carbon phases, and so, the line position is marked in black in the maps of Figs. 2(b) and 2(c) by overlapping the region where the peak intensity is below 20\%. In the region between the two lines, a small homogeneous broadening of the Raman

TABLE I. Selection rules-backscattering from (110).

\begin{tabular}{lcc}
\hline \hline Geometry & Component $/ /[001]$ & Component $/ /[\overline{1} 10]$ \\
\hline $\bar{X}^{\prime}\left(\mathrm{Y}^{\prime} \mathrm{Y}^{\prime}\right) \mathrm{X}^{\prime}$ & $\checkmark$ & $\cdots$ \\
$\bar{X}^{\prime}\left(\mathrm{Y}^{\prime} \mathrm{Z}^{\prime}\right) \mathrm{X}^{\prime}$ & $\ldots$ & $\checkmark$ \\
$\bar{X}^{\prime}\left(\mathrm{Z}^{\prime} \mathrm{Y}^{\prime}\right) \mathrm{X}^{\prime}$ & $\cdots$ & $\checkmark$ \\
$\bar{X}^{\prime}\left(\mathrm{Z}^{\prime} \mathrm{Z}^{\prime}\right) \mathrm{X}^{\prime}$ & $\cdots$ & $\cdots$ \\
\hline \hline
\end{tabular}
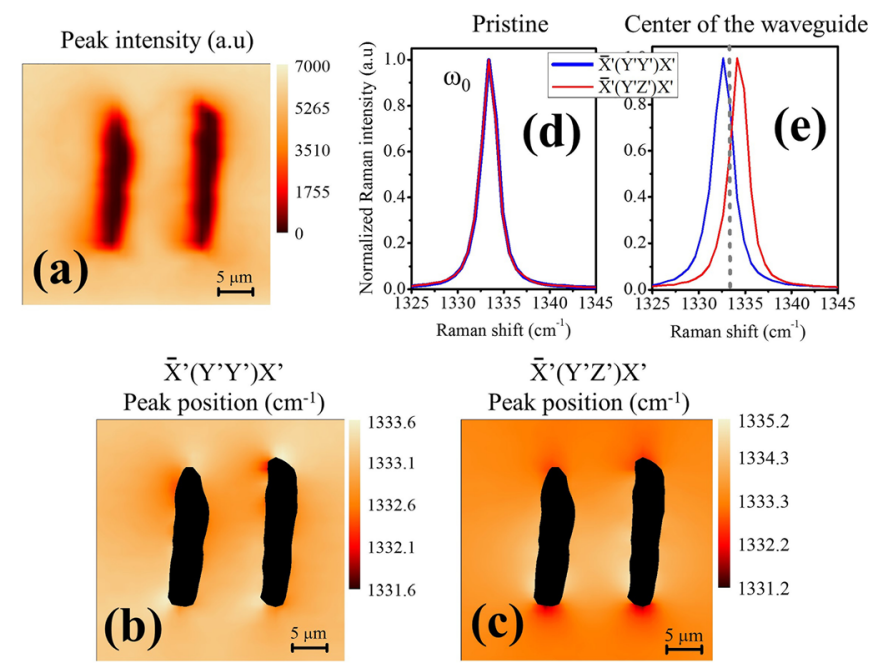

FIG. 2. Maps of the (a) intensity and the shift of the diamond Raman peak in the (b) $\bar{X}^{\prime}\left(Y^{\prime} Y^{\prime}\right) X^{\prime}$ and (c) $\bar{X}^{\prime}\left(Y^{\prime} Z^{\prime}\right) X^{\prime}$ configurations, obtained from the fit of the spectra to Lorentzian curves (scanning step of $1 \mu \mathrm{m}$ ). Raman spectra recorded (d) outside and (e) within the waveguide region.

peak from a width of $2.1 \mathrm{~cm}^{-1}$ to a width of $2.4 \mathrm{~cm}^{-1}$ is detected, related to the presence of stress. ${ }^{35,36}$ The linewidth is similar along the guiding region, which is an indication of fairly uniform stress distribution.

Following the selection rules of Table I, if the configuration $\bar{X}^{\prime}\left(\mathrm{Y}^{\prime} \mathrm{Y}^{\prime}\right) \mathrm{X}^{\prime}$ is selected, the component parallel to [001] can be obtained and is the one presented in Fig. 2(b). On the other hand, the $\bar{X}^{\prime}\left(\mathrm{Y}^{\prime} \mathrm{Z}^{\prime}\right) \mathrm{X}^{\prime}$ configuration is shown in Fig. 2(c), which is associated with the component parallel to [110]. The shift of these two components [Figs. 2(d) and $2(\mathrm{e})]$ is a first indication that we can have stress with different character (tensile or compressive) in the [001] and [1 10] directions. In order to calculate the stress responsible for these shifts, Eqs. (6) and (7) can be applied. A equation system must be solved in order to extract the values of $\tau_{1}$ and $\tau_{2}$ from the measured shifts

$$
\tau_{1}=-\frac{\Delta \omega_{2}-0.53 \Delta \omega_{3}}{1.85} ; \quad \tau_{2}=-\frac{\Delta \omega_{3}+0.82 \tau_{1}}{1.55} .
$$

At this point, we have to consider the effect that the two laser lines can have on the surrounding material. The laser causes the amorphization of the crystal lattice and the transformation of $\mathrm{sp}^{3}$-diamond bonding into $\mathrm{sp}^{2}$ bondings. ${ }^{10}$ This transformation generates a material with lower density inside the lines, producing a local volume expansion. Furthermore, the stress produced in the $[\overline{1} 10]$ direction $\left(\tau_{1}\right)$ will be compressive, whereas in the perpendicular direction, there will be a shear tensile stress $\left(\tau_{2}\right)$. Taking this into account, we associate the correct components $\Delta \omega_{2}$ and $\Delta \omega_{3}$ with the measured Raman components, and the calculated stress for each point in the map is shown in Figs. 3(a) and 3(b). In these maps, it can be observed that $\tau_{1}$ has a compressive character between the two lines, whereas $\tau_{2}$ is mainly tensile. The sign of both stresses is inverted at the top and bottom tips of the lines (in a similar way to the theoretical study covering lithium niobate ${ }^{18}$ ). From the vertical line profile of both stresses $\tau_{1}$ and $\tau_{2}$ measured at the center of the waveguide [Fig. 3(c)], it can be observed that the maximum value for both is located near the center of the waveguide. 

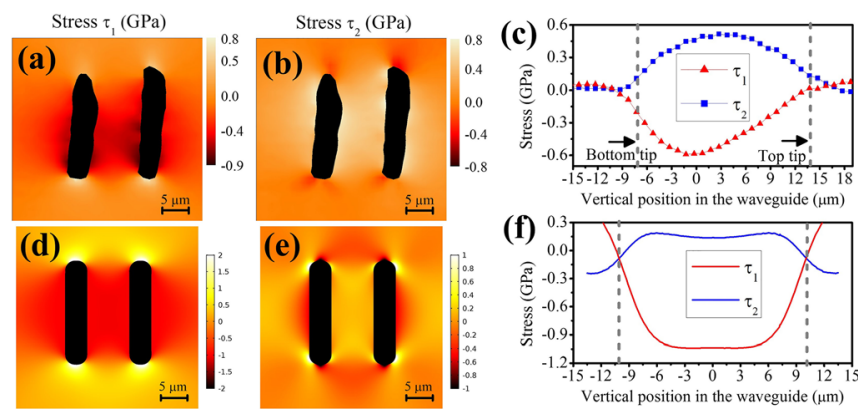

FIG. 3. Maps of the $\tau_{1}$ and $\tau_{2}$ induced stresses in the waveguide: (a) and (b) from the Raman peak shift and (d) and (e) from 2D Finite Element simulations. Vertical line profile of $\tau_{1}$ and $\tau_{2}$ at the center of the waveguide: (c) measured and (f) simulated.

The calculated stresses are compared with those obtained from 2D Finite Element simulations of the constrained expansion of two amorphous carbon inclusions in a diamond matrix. The adopted procedure is described in the studies by Bosia et al. ${ }^{37}$ and Battiato et al. ${ }^{38}$ To approximate the real geometry, two parallel rectangles with filleted corners are considered. The simulated $\tau_{1}$ and $\tau_{2}$ stress distributions, shown in Figs. 3(d)-3(f), correctly reproduce those observed experimentally. The small discrepancies and the asymmetry between the curves of $\tau_{1}$ and $\tau_{2}$ in Fig. 3(c) can be attributed to the simplified geometry in the simulations and possible inhomogeneities in material properties in the amorphized regions.

Once the stresses have been calculated from the measured shift of the Raman peak, the change in the refractive index can be obtained following the same strategy as suggested by Burghoff et al. ${ }^{18}$ Stress values are related to the refractive index through the piezooptic tensor $\pi_{i j}$

$$
\Delta\left(\frac{1}{n^{2}}\right)_{i}=\pi_{i j} \sigma_{j}
$$

As for the compliance tensor, due to the symmetry of the diamond crystal structure, only three components of the piezooptic tensor are non-zero: $\pi_{11}=-0.43 \times 10^{-12} \mathrm{~Pa} ; \pi_{12}$ $=0.37 \times 10^{-12} \mathrm{~Pa} ; \pi_{44}=-0.27 \times 10^{-12} \mathrm{~Pa}^{32}$ Previous experimental studies have shown that tensile hydrostatic pressure produces an increase in the refractive index of diamond. ${ }^{39-41}$ This effect is related to an increment of the electronic polarizability overbalancing the reduction of the density ${ }^{39}$ and has been observed in other materials, such as $\mathrm{MgO}, \mathrm{SiC}$, or sapphire. ${ }^{39,41,42}$ Here, we have a biaxial stress, and so the different components of the $\Delta\left(1 / n^{2}\right)_{i}$ tensor must be calculated to assess the character of the refractive index change. Using Eq. (9) and the values of stress $\tau_{1}$ and $\tau_{2}$, we can obtain the refractive index profile across the waveguide for the polarization parallel to [001] [Fig. 4(a)]. A well localized region of increased refractive index is visible. Comparing the map of the refractive index change with the image of an optical mode [Fig. 4(c)], we can see that the mode is located at the same position as the increased refractive index region. For the polarization parallel to $[\overline{1} 10]$, the refractive index decreases in the guiding region.

From the map in Fig. 4(a), we can see that the optical mode is confined in the horizontal direction by the presence

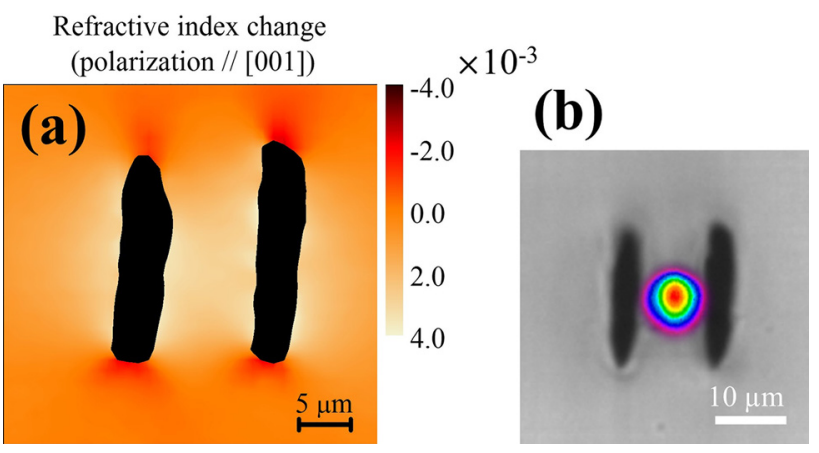

FIG. 4. (a) Map of the refractive index profile. (b) Optical microscopy image with the overlaid optical TM mode measured using a beam-profiler (mode field diameter of $\sim 10 \mu \mathrm{m}$ ).

of the two laser-written lines which act as barriers due to the decrease in $n$ expected in these regions. In the vertical direction, light is confined due to the stress-induced change in the refractive index. At the center of the waveguide, $\Delta n$ is calculated to be $3 \times 10^{-3}$.

Finally, from the results we have obtained, we can gain a deeper understanding of the polarization behavior of the waveguides. We have reported before that the TM mode is guided, whereas the TE is not. ${ }^{10}$ The TM mode will have the polarization parallel to the [001] crystallographic direction in the waveguides. So, this polarization experiences an increase in the refractive index within the central region between the two barriers [Fig. 4(a)], and the mode is confined. On the other hand, the polarization parallel to $[\overline{1} 10]$ sees a decrease in the refractive index, and thus, the TE mode does not meet the conditions to be guided.

In conclusion, we have described the use of polarized Raman spectroscopy to obtain information about the stress distribution and refractive index change in diamond waveguides fabricated with femtosecond laser writing. We have shown that the optical mode is confined in the horizontal direction by the two laser-written lines, whereas in the vertical direction, it is confined by the stress-induced refractive index change. The polarization behavior of the waveguides is explained using the stress created in the guiding region. This study provides a useful framework to design the properties of the diamond waveguides by changing the femtosecond laser writing parameters. The method can be extended to analyze stress-induced femtosecond laser written waveguides with different geometries in diamond as well as in other crystals.

The authors acknowledge support from the FP7 DiamondFab CONCERT Japan project, the DIAMANTE MIUR-SIR Grant, and the FemtoDiamante Cariplo ERC reinforcement grant. We thank Professor Guglielmo Lanzani and Dr. Luigino Criante for the use of the FemtoFab facility at CNST-IIT Milano for the laser fabrication experiments. We thank Professor Roberto Osellame for access to waveguide characterization facilities. We thank Dr. Patrick Salter for helpful scientific discussions.

${ }^{1}$ I. Aharonovich, S. Castelletto, D. A. Simpson, C. H. Su, A. D. Greentree, and S. Prawer, Rep. Prog. Phys. 74, 076501 (2011).

${ }^{2}$ P. Neumann, I. Jakobi, F. Dolde, C. Burk, R. Reuter, G. Waldherr, J. Honert, T. Wolf, A. Brunner, J. H. Shim et al., Nano Lett. 13, 2738-2742 (2013). 
${ }^{3}$ C. T. Nguyen, R. E. Evans, A. Sipahigil, M. K. Bhaskar, D. D. Sukachev, V. N. Agafonov, V. A. Davydov, L. F. Kulikova, F. Jelezko, and M. D. Lukin, preprint arXiv:1708.05419 (2017).

${ }^{4}$ R. Schirhagl, K. Chang, M. Loretz, and C. L. Degen, Annu. Rev. Phys. Chem. 65, 83-105 (2014).

${ }^{5}$ I. Aharonovich, A. D. Greentree, and S. Prawer, Nat. Photonics 5, 397-405 (2011).

${ }^{6}$ P. Olivero, S. Rubanov, P. Reichart, B. C. Gibson, S. T. Huntington, J. Rabeau, A. D. Greentree, J. Salzman, D. Moore, D. N. Jamieson et al., Adv. Mater. 17, 2427-2430 (2005).

${ }^{7}$ B. Khanaliloo, H. Jayakumar, A. C. Hryciw, D. P. Lake, H. Kaviani, and P. E. Barclay, Phys. Rev. X 5, 041051 (2015).

${ }^{8}$ M. J. Burek, N. P. de Leon, B. J. Shields, B. J. Hausmann, Y. Chu, Q. Quan, A. S. Zibrov, H. Park, M. D. Lukin, and M. Lončar, Nano Lett. 12, 6084-6089 (2012).

${ }^{9}$ S. Lagomarsino, P. Olivero, F. Bosia, M. Vannoni, S. Calusi, L. Giuntini, and M. Massi, Phys. Rev. Lett. 105, 233903 (2010).

${ }^{10}$ B. Sotillo, V. Bharadwaj, J. P. Hadden, M. Sakakura, A. Chiappini, T. T. Fernandez, S. Longhi, O. Jedrkiewicz, Y. Shimotsuma, L. Criante, R. Osellame, G. Galzerano, M. Ferrari, K. Miura, R. Ramponi, P. E. Barclay, and S. M. Eaton, Sci. Rep. 6, 35566 (2016).

${ }^{11}$ A. Courvoisier, M. J. Booth, and P. S. Salter, Appl. Phys. Lett. 109, 031109 (2016).

${ }^{12}$ V. Bharadwaj, A. Courvoisier, T. T. Fernandez, R. Ramponi, G. Galzerano, J. Nunn, M. J. Booth, R. Osellame, S. M. Eaton, and P. S. Salter, Opt. Lett. 42, 3451-3453 (2017).

${ }^{13}$ J. P. Hadden, V. Bharadwaj, B. Sotillo, S. Rampini, R. Osellame, R. J. Witmer, H. Jayakumar, T. T. Fernandez, A. Chiappini, C. Armellini, M. Ferrari, R. Ramponi, P. E. Barclay, and S. M. Eaton, preprint arXiv:1701.05885 (2017).

${ }^{14}$ K. M. Davis, K. Miura, N. Sugimoto, and K. Hirao, Opt. Lett. 21, 1729-1731 (1996).

${ }^{15}$ S. M. Eaton, H. Zhang, P. R. Herman, F. Yoshino, L. Shah, J. Bovatsek, and A. Y. Arai, Opt. Express 13, 4708-4716 (2005).

${ }^{16}$ R. R. Gattass and E. Mazur, Nat. Photonics 2, 219-225 (2008).

${ }^{17}$ F. Chen and J. R. Aldana, Laser Photonics Rev. 8, 251-275 (2014).

${ }^{18}$ J. Burghoff, S. Nolte, and A. Tünnermann, Appl. Phys. A 89, 127-132 (2007).

${ }^{19}$ S. M. Eaton, C. A. Merchant, R. Iyer, A. Zilkie, A. S. Helmy, J. S. Aitchison, P. R. Herman, D. Kraemer, R. J. D. Miller, C. Hnatovsky et al., Appl. Phys. Lett. 92, 081105 (2008).

${ }^{20}$ A. Ródenas, L. M. Maestro, M. O. Ramírez, G. A. Torchia, L. Roso, F. Chen, and D. Jaque, J. Appl. Phys. 106, 013110 (2009).
${ }^{21}$ A. Benayas, W. F. Silva, A. Ródenas, C. Jacinto, J. V. de Aldana, F. Chen, Y. Tan, R. R. Thomsom, N. D. Psaila, D. T. Reid, G. A. Torchia, A. K. Kar, and D. Jaque, Appl. Phys. A 104, 301-309 (2011).

${ }^{22}$ P. Oberson, B. Gisin, B. Huttner, and N. Gisin, Appl. Opt. 37, 7268-7272 (1998).

${ }^{23}$ S. M. Eaton, M. L. Ng, R. Osellame, and P. R. Herman, J. Non-Cryst. Solids 357, 2387-2391 (2011).

${ }^{24}$ P. Ferraro, L. Miccio, S. Grilli, M. Paturzo, S. De Nicola, A. Finizio, R. Osellame, and P. Laporta, Opt. Express 15, 14591-14600 (2007).

${ }^{25}$ R. Mildren and J. Rabeau, Optical Engineering of Diamond (John Wiley \& Sons, 2013).

${ }^{26}$ S. Ganesan, A. A. Maradudin, and J. Oitmaa, Ann. Phys. 56, 556-594 (1970).

${ }^{27}$ M. H. Grimsditch, E. Anastassakis, and M. Cardona, Phys. Rev. B 18, 901 (1978).

${ }^{28}$ J. W. Ager III and M. D. Drory, Phys. Rev. B 48, 2601 (1993).

${ }^{29}$ Y. Von Kaenel, J. Stiegler, J. Michler, and E. Blank, J. Appl. Phys. 81, 1726-1736 (1997).

${ }^{30}$ E. Anastassakis, J. Appl. Phys. 86, 249-258 (1999).

${ }^{31}$ T. Gries, L. Vandenbulcke, P. Simon, and A. Canizares, J. Appl. Phys. 102, 083519 (2007).

${ }^{32}$ J. F. Nye, Physical Properties of Crystals: Their Representation by Tensors and Matrices (Oxford University Press, 1985).

${ }^{33}$ E. Anastassakis, A. Pinczuk, E. Burstein, F. H. Pollak, and M. Cardona, Solid State Commun. 8, 133-138 (1970).

${ }^{34}$ C. A. Arguello, D. L. Rousseau, and S. P. S. Porto, Phys. Rev. 181, 1351 (1969).

${ }^{35}$ L. Bergman and R. Nemanich, J. Appl. Phys. 78, 6709-6719 (1995).

${ }^{36} \mathrm{R}$. Erasmus, J. Comins, V. Mofokeng, and Z. Martin, Diamond Relat. Mater. 20, 907-911 (2011).

${ }^{37}$ F. Bosia, N. Argiolas, M. Bazzan, B. A. Fairchild, A. D. Greentree, D. W. Lau, P. Olivero, F. Picollo, S. Rubanov, and S. Prawer, J. Phys.: Condens. Matter 25, 385403 (2013).

${ }^{38}$ A. Battiato, M. Lorusso, E. Bernardi, F. Picollo, F. Bosia, D. Ugues, A. Zelferino, A. Damin, J. Baima, N. M. Pugno et al., Acta Mater. 116, 95-103 (2016).

${ }^{39}$ R. M. Waxler and C. E. Weir, J. Res. Natl. Bur. Stand., Sect. A 69A, 325 (1965).

${ }^{40}$ J. Fontanella, R. L. Johnston, J. H. Colwell, and C. Andeen, Appl. Opt. 16, 2949-2951 (1977).

${ }^{41}$ N. M. Balzaretti and J. A. H. Da Jornada, Solid State Commun. 99, 943-948 (1996).

${ }^{42}$ S. C. Jones, B. A. M. Vaughan, and Y. M. Gupta, J. Appl. Phys. 90, 4990-4996 (2001). 